\title{
CONTROVERSIES IN FLUID MANAGEMENT AND EVIDENCE BASED THERAPEUTIC IMPLICATIONS
}

\author{
E.H. Mithrajee Jayadeesha Premaratne* \\ Acting Consultant in Anaesthesiology- Base Hospital Balapitiya- Sri Lanka. \\ *Corresponding author's e-mail: mithrajee1@yahoo.co.uk
}

Key words- Crystalloids vs colloids, fluid management in sepsis, acute lung injury, trauma

\section{Crystalloids versus Colloids}

The Saline versus Albumin Fluid Evaluation [SAFE] Study ${ }^{1}$ debunks 1998 Cochrane Injuries Group Albumin Reviewers meta-analysis ${ }^{2}$, associating albumin with increased mortality in critical care populations. There was no significant difference in survival between those who receive either $4 \%$ albumin or normal saline. SAFE study ${ }^{1,3}$ has shown that baseline albumin concentration had no impact on the treatment effect even after correction for other baseline risk factors. From 2003 Cochrane review there is no evidence to support the superiority of any particular colloid over others. ${ }^{4}$

\section{Evidence based therapeutic implications;}

1. Crystalloids should be administered first in non-hemorrhagic shock resuscitation. ${ }^{1}$

2. Although albumin does not increase the risk of mortality in patients with hypoalbuminaemia, data do not support its routine use to maintain or increase intravascular volume in critically ill adults. ${ }^{3}$

3. Hydroxyethyl starch solutions should be used with caution in cardiopulmonary bypass. There is conflicting evidence that HES increases the risk of bleeding after cardiopulmonary bypass surgery. ${ }^{5}$

4. Limited evidence (albeit not statistically significant) of a treatment effect that favors albumin in patients with severe sepsis. ${ }^{1}$

5. HES administration may increase the risk of acute renal failure in patients with sepsis. ${ }^{5}$

6. Colloids are preferred for treating dialysisassociated hypotension and in maintaining hemodynamics to achieve dialysis goals. ${ }^{5}$

7. There is no evidence of a benefit of colloids in treating ischemic brain injury or subarachnoid hemorrhage. Colloids may adversely impact survival in traumatic brain injury
8. Hyperoncotic albumin should be administered in conjunction with large-volume paracentesis for diuretic-refractory ascites (II-A).

9. Albumin may be administered in conjunction with antimicrobial therapy to patients with spontaneous bacterial peritonitis (II-A).

\section{Fluid resuscitation in sepsis}

Early goal-directed therapy provides significant benefits with respect to outcome in patients with severe sepsis and septic shock. For every 7 patients with severe sepsis or septic shock treated with early goal-directed therapy, compared to conventional therapy, one less patient dies in hospital. ${ }^{6}$

\section{Evidence based therapeutic implications.}

1. Initial Resuscitation ${ }^{7}$ of a patient having severe sepsis or sepsis-induced tissue hypoperfusion should begin as soon as the syndrome is recognized and should not be delayed pending intensive care unit (ICU) admission.

2. Fluid resuscitation may consist of natural or artificial colloids or crystalloids. There is no evidence-based support for one type of fluid over another.

3. Patients should receive the initial minimum $20 \mathrm{~mL} / \mathrm{Kg}$ fluid challenge prior to placement of a central venous catheter and attempts to maximize CVP.

4. Fluid challenge in patients with suspected hypovolemia may be given at a rate of 500$1000 \mathrm{~mL}$ of crystalloids or $300-500 \mathrm{~mL}$ of colloids over 30 mins and repeated based on response.

5. During the first $6 \mathrm{hrs}$ of resuscitation, the goals of initial resuscitation of sepsis-induced hypoperfusion should include all of the following as one part of a treatment protocol: ${ }^{7}$ $\square$ Central venous pressure: 8-12 mm Hg $\square$ Mean arterial pressure $>65 \mathrm{~mm} \mathrm{Hg}$ 
$\square$ Urine output $>0.5 \mathrm{~mL} / \mathrm{kg} / \mathrm{hr}$

$\square$ Central venous (superior vena cava) or mixed venous oxygen saturation $>70 \%$.

\section{Fluid-management strategies in Acute Lung Injury}

Fluids and Catheters Treatment Trial (FACTT) was carried out to determine whether a conservative or a liberal strategy of fluid management was more effective in patients with established acute lung injury. ${ }^{8}$ Although there was no difference in mortality, in the group treated according to a conservative strategy of fluid management had significantly improved lung function [the oxygenation index and the lung injury score ] and CNS function and a decreased need for sedation, mechanical ventilation, and intensive care without increase in the frequency of non pulmonary organ failure or shock.

\section{Evidence based Therapeutic Implications}

1. Fluid restriction is appropriate for patients with hemodynamically stable ALI/ARDS. Conservative fluid management during the established phase of acute lung injury is just as important as titrated liberal administration during the acute phase of the inciting insult

2. The combination of colloids and diuretics may be considered in patients with hypo-oncotic ALI/ARDS. ${ }^{5}$

\section{Fluid-management strategies of trauma victims.}

Early fluid resuscitation has traditionally been used to avert shock and its attendant complications, yet clinical data suggest that definitive bleeding control is imperative before aggressive asanguineous resuscitation ${ }^{9}$. According to the recent Cochrane Systematic Review ${ }^{10}$ there is no evidence for or against early or larger volume of intravenous fluid administration in uncontrolled haemorrhage. There is continuing uncertainty about the best fluid administration strategy in bleeding trauma patients. Further randomised controlled trials are needed to establish the most effective fluid resuscitation strategy.

\section{Evidence based therapeutic implications.}

1. Cannulation should take place en route where possible. ${ }^{12}$

2. Transfer should not be delayed by attempts to obtain intravenous access. ${ }^{12}$

3. Entrapped patients require cannulation at the scene ${ }^{12}$
4. Normal saline is recommended as a suitable fluid for administration to trauma patients. ${ }^{1}$

5. Boluses of $250 \mathrm{ml}$ fluid may be titrated against the presence or absence of a radial pulse (caveats; penetrating torso injury, head injury, infants) ${ }^{12}$

6. Colloids should be avoided or used with caution in patients with traumatic brain injury. ${ }^{1,13}$

\section{Conclusion}

Fluid may be a friend when appropriately titrated during the resuscitation. However, excess fluid becomes an enemy when it is no longer physiologically needed. According to the current state of knowledge, factors that may influence the choice of resuscitation fluid for critically ill patients include the patient-specific conditions, clinician's preference, the tolerability of the treatment, its safety, and its cost.

\section{References;}

1. Finfer S, Bellomo R, Boyce N, French J, Myburgh J, Norton R .The SAFE Study Investigators. A comparison of albumin and saline for fluid resuscitation in the intensive care unit. $\mathrm{N}$ Engl $\mathrm{J}$ Med 2004;350:2247-2256

2. Cochrane Injuries Group Albumin Reviewers. Human albumin administration in critically ill patients: systematic review of randomised controlled trials. BMJ 1998;317:235-240.

3. Effect of baseline serum albumin concentration on outcome of resuscitation with albumin or saline in patients in intensive care units: analysis of data from the saline versus albumin fluid evaluation (SAFE) study - BMJ 2006;333:1044

4. Bunn F, Alderson P, Hawkins V Cochrane Database Syst Rev. 2003; (1):CD001319

5. American Thoracic Society Consensus Statement American Journal of Respiratory and Critical Care Medicine Vol 170. pp. 1247-1259, (2004)

6. Early Goal-Directed Therapy in the Treatment of Severe Sepsis and Septic Shock.Rivers E, Nguyen B, Havstad S, et al. Early goal-directed therapy in the treatment of severe sepsis and septic shock. NEJM. 2001 Nov 8, Number 19;345:1368-1377.

7. Surviving sepsis campaign guidelines for management of severe sepsis and septic shock. Crit Care Med 2004 Mar;32(3):858-73

8. Comparison of Two Fluid-Management Strategies in Acute Lung Injury Fluids and Catheters Treatment Trial (FACTT) The National Heart, Lung, and Blood Institute Acute Respiratory Distress Syndrome (ARDS) Clinical Trials Network NEJM- 354:2564-2575

9. Bickell WH, Wall MJ, Pepe PE, Martin RR, Ginger VF, Allen MK, Mattox KL. Immediate versus delayed fluid resuscitation for hypotensive patients 
with penetrating truncal injuries. $N$ Engl $\mathrm{J}$ Med 1994;331

10. Kwan I, Bunn F, Roberts I, on behalf of the WHO Pre-Hospital Trauma Care Steering Committee.Timing and volume of fluid administration for patients with bleeding. Cochrane Database of Systematic Reviews 2003, Issue 3.

11. Saline or Albumin for Fluid Resuscitation in Patients with Traumatic Brain Injury, N Engl J Med 2007;357:874-84.
12. Fluid resuscitation in prehospital trauma care: a consensus view M Revell, K Porter, I Greaves Emerg Med J 2002;19: 494-498.

13. Saline or Albumin for Fluid Resuscitation in Patients with Traumatic Brain Injury, N Engl J Med 2007;357:874-84.

\section{THE COLLEGE OF ANAESTHESIOLOGISTS OF SRI LANKA}

\section{B S PERERA ORATION}

Applications are hereby called for the "B. S. Perera Oration", to be held on the $23^{\text {rd }}$ January 2010 at the $26^{\text {th }}$ Annual Scientific Sessions of the college.

Terms and Conditions for the Oration.

1. Five copies of the entire oration should be sent before $31^{\text {st }}$ October 2009 to The Secretary, College of Anaesthesiologists of Sri Lanka and there should not be any substantial alteration in the text when the lecture is finally delivered.

2. The lectureship is open to all members of the College of Anaesthesiologists.

3. Five copies of a brief resume of the salient points of the paper should also be submitted indicating any contribution to further advances in knowledge of the subject.

4. The lecture should preferably not exceed 45 minutes.

5. A committee of five will be nominated by the council to scrutinize papers submitted and to select a suitable candidate. This committee of five shall award the lectureship.

6. The lecture should be related to anaesthesia and intensive care and should be based on the original work of the lecturer.

7. In the event of the committee being unable to select a lecturer from the applicants, they may invite a lecturer of international repute to deliver the lecture ship.

8. The honorium may be in the form of a gold medal.

9. Press should not be invited and photographs and accounts of the lecture should not be published in the Newspapers, without the permission of the College Council.

10. Copyright of the oration belongs to the College of Anaesthesiologists of Sri Lanka. 